\title{
VISIÓN DE ENSEÑANZA Y APRENDIZAJE \\ DE LA CIENCIA DESDE DOS PROFESORES \\ DE QUÍMICA
}

\author{
Yolanda Ladino Ospina \\ Profesora. Estudiante doctorado en Educación en Ciencias. Universidad \\ Pedagógica Nacional. \\ Rigoberto Gómez Cruz \\ Profesor. Universidad Pedagógica Nacional.
}

\begin{abstract}
In the educational environment, the qualitative methods of research are seen as an option in the classroom for unveiling the teachers' conceptions when they are the "actors" in the teaching learning of sciences process. The results shown were collected using a study case methodology. The purpose is not to criticize the two science teacher's rol, nor even their docent action, en the contrary, this is a reflection about the different knowledges, methods and techniques employed by the teachers in the classroom.
\end{abstract}

\section{RESUMEN}

En el ámbito educativo, los métodos de investigación cualitativa, son vistos como una alternativa en el aula de clase para describir las actitudes y las concepciones de los profesores cuando son "actores" no circunstanciales del proceso de enseñanza y aprendizaje de las ciencias. Los resultados que a continuación se presentan se recolectaron empleando una metodología de estudio de caso. No se pretende criticar la actuación de los dos profesores de ciencias, ni mucho menos cuestionar su acción docente, solo es una reflexión, una aproximación a los diferentes saberes, métodos y técnicas que emplean los maestros en el aula de clase.

\section{INTRODUCCIÓN}

Varios son los campos de aplicación de la metodología de estudio de caso: la industria, la producción, el mercadeo, la psicología y en nuestro caso particular en el ambiente educativo; como lo menciona Stenhouse, "el estudio de caso, involucra la colección de varios datos, la preparación y la presentación de un reporte" (Stenhouse, 1990, p. 49); aquí es visto como una investigación, que es específica, interesante y no se revierte en generalizaciones, sino justamente, pretende revelar una actuación y jo representación personal de dos profesores que se desenvuelven en el ámbito escolar. 


\section{METODOLOGIA}

La metodología de estudio de caso pertenece a los métodos de investigación cualitativa, que requieren especial atención, particularmente en cuanto a establecer la relación entre el investigador y lo investigado (Smith, 1983, p. 8). Hay varias formas diferentes de recolectar datos según la perspectiva de la investigación; sin embargo, es importante recordar que la técnica sea clara y apropiada para recolectar datos. En términos de Martínez, un estudio de caso implica una fase preactiva o de preparación de la investigación, una intermedia o interactiva, que coincide con el trabajo de campo y la recolección de los datos y una tercera o posactiva que incluye la elaboración del reporte final. Según el mismo autor, la forma de recolección de datos, incluye métodos de lápiz y papel que emplean técnicas como las notas de campo del profesor, el diario de los alumnos y los cuestionarios; métodos interactivos, donde se prefieren técnicas sociométricas, interacciones, entrevistas y discusiones (Martínez B. Jaume, 1988), y recientemente se han incorporado otras formas como la toma de fotos, las grabaciones $\mathrm{y}$ el video, que es el fundamento de la recolección de datos en este trabajo.

La presente investigación, consiste en un estudio de caso sobre la concepción de enseñanza y aprendizaje que tienen dos profesores de química y además, reflejan en su quehacer diario. Para ello se recurrió a las técnicas de entrevista, filmación de las clases de los profesores colaboradores y la triangulación de la investigación que se realizó cuando el profesor observó la grabación de su clase, escuchó la entrevista y al final realizó una interpretación sobre su actuación personal (Martínez B. Jaume, 1988.).

\section{RESULTADOS}

Para no entrar en especulaciones, solo diremos que el profesor A, tiene una formación inicial de Licenciado en Química, con maestría en Docencia de la Química y especialización en Historia y Epistemología de las Ciencias y el Profesor B, tiene estudios profesionales en Química y su área de formación es la química.

Después de emplear las tres técnicas para recolección de datos sobre los profesores $A$ y $B$, se identifican patrones de acción e interacción que permiten establecer algunas categorías para analizar los datos y presentar este reporte. Para analizar la información recolectada, se identificaron como categorías principales: la concepción de ciencia de cada profesor, la concepción de enseñanza y aprendizaje, la coherencia entre el pensamiento, la actuación y el rol del profesor en el aula de clase. (Guerrero, 1987, Fernández y Elortegui, 1996 y Reyes y otros, 1999).

En cuanto a la concepción de ciencia que cada profesor tiene y refleja a través de la entrevista, encontramos que esta depende mucho de la formación inicial de cada profesor. Para el profesor A:

"La ciencia o las ciencias es uno de esos conceptos que tercamente se resisten a ser definidos, máxime cuando para las personas como nosotros los docentes que estamos en constante intercambio, diría yo, de información en constante diálogo con profesores que hacen parte de la comunidad científica, lo que uno pues, encuentra es que concepciones de ciencia parece que hay muchas y muy diversas, realmente la discusión epistemológica contemporánea sobre la ciencia y el desarrollo de la ciencia, pues ha mostrado que existen diferentes concepciones sobre lo que ya es, sin embargo yo diría que la ciencia es una actividad racional que realizan los seres 
humanos, porque creo que no existe otra especie por lo menos conocida que sea capaz de hacer ciencia";

mientras para el profesor B, la ciencia es más entendida como:

"un conjunto, una parte de conocimientos específicos en un área y fundamentalmente de métodos de cómo se adquiere ese conocimiento en el área correspondiente, a diferencia de otros entornes en donde se puede producir conocimiento, la ciencia tiene unos modelos que son mucho más precisos".

Las dos concepciones son realmente justificadas por el pensamiento y la actuación del profesor, en el aula de clase. El profesor A, según lo muestra la filmación, es muy espontáneo y sistemático, busca a partir de la actividad racional de los alumnos, construir conocimiento en el aula de clase, así como lo ratifica una vez él analiza su actuación en el video. El profesor B (visto en el vídeo e interpretado por él mismo posteriormente), busca que en las clases de ciencias, los alumnos no tomen los conceptos como verdades absolutas, busca, e inclusive logra en sus clases, crear un ambiente de diálogo y confrontación teórica, práctica y matemática de los conceptos involucrados, siempre en procura de nuevas relaciones y nuevas evidencias conceptuales.

Esta visión anterior refuerza el planteamiento de cada profesor frente a las implicaciones de lo que se entiende por ciencia, en el ámbito escolar y social de nuestro entorno educativo en particular. Por ejemplo, para el profesor A,

"es una forma también de acceder, bueno no de acceder de construir conocimiento, como otras actividades, lo mismo que el arte, la religión, etc., también son formas de conocimiento válidas, que son igualmente aceptadas y algunos se dedican a trabajar en ciencias, otras no, otras se dedican a la religión al arte, lo que pasa es que en nuestra sociedad, se la ha dado un estatus a lo que se denomina científico".

De igual manera el profesor $\mathrm{B}$, analiza las implicaciones que tiene la ciencia y la contribución o no al ambiente escolar, así como también establece los límites y fronteras de generación y aplicación del conocimiento particular de las ciencias,

"Como base de todo conocimiento científico, siempre está un modelo matemático y aceptamos esa lógica formal matemática, pues tiene herramientas, que nos permiten con precisión pronosticar cierto tipo de eventos eso nos distingue, por ejemplo, de las ciencias sociales en las cuales si bien, yo puedo asimilar algunos de los conocimientos matemáticos, el exigir la completa predictibilidad que puede dar eso es muy relativo yo tengo una concordancia completa entre un modelo propuesto de manera teórica con base en la lógica matemática y el comportamiento real de los sistemas, que es desafortunadamente lo que la mayoría de las personas no ven, la mayoría de personas ven que si es algo que se establece mediante un modelo científico entonces tiene que ser cierto como una verdad absoluta, yo siempre critico desde ese punto de vista a los matemáticos que se comportan como si hubiesen descubierto a Dios, si, para ellos las matemáticas son el principio y el fin de todas las cosas, no son solamente herramientas que me sirven y los casos reales se semejan en un comportamiento límite a esos modelos, entonces el conocimiento científico pues busca confirmar los modelos que funcionan en gran cantidad de aplicaciones".

Las dos posturas anteriores, permiten inferir primero, que no hay una sola concepción de ciencia, que según la formación profesional inicial de cada profesor, la ciencia es vista 
con mucha o menos rigurosidad, pero ambas posturas admiten la presencia de otras formas de conocimiento y las variables que en dichas formas de conocimiento se pueden presentar.

En cuanto a la segunda categoría analizada, la concepción de enseñanza y aprendizaje, se puede decir que para ambos profesores, uno de sus objetivos finales en el aula de clase es que los alumnos adquieran conocimientos que tengan significancia y coherencia para ellos, esto se revela, no sólo en la entrevista, el video y el análisis posterior, ya que, cada profesor da un papel protagónico a lo que el estudiante está pensando y ese es un punto de partida muy importante para la enseñanza y el aprendizaje, donde se relacionan aspectos teóricos con aspectos prácticos de la ciencia y de la vida diaria; así, para el profesor A,

"Lo más importante en el proceso de enseñanza y aprendizaje no tanto es que el profesor muestre que tanto, que tan bien domina un tema y lo organiza, sino que además fundamentalmente me preocupan los niveles de aprendizaje que alcancen los estudiantes, entonces yo diría que en este sentido, soy un profesor que maneja mucho estilos de enseñanza basados en el alumno, en este sentido pues, trabajo mucho con técnicas grupales, donde los estudiantes tengan la oportunidad de discutir temas centrales, presentar sus propias ideas, argumentar, sí, es decir, darle muchas mas responsabilidad a los estudiantes en la preparación de las temáticas".

Esto último, se observa en el vídeo de la clase, donde el profesor después de desarrollar un aspecto teórico, cuestiona a los alumnos sobre las relaciones y variables que se implican, por ejemplo en la estequiometría de la reacción del $\mathrm{Zn}$ con el $\mathrm{HCl}$, los alumnos discuten e intercambian ideas en grupo y luego realizan una puesta en común. Así mismo, el profesor $B$, que en sus clases lo que importa es lo que sus alumnos estén en capacidad de desarrollar, se encuentra que:

"Continuamente trato de hacer, de dar la motivación, de incitar a los estudiantes a que hagan lo que no hicieron los profesores conmigo, es decir, yo reconozco que los profesores que tuve durante mi formación, se limitaron únicamente a dar una serie de conocimientos de contenidos establecidos, pero más allá no había ningún ambiente para dar rienda suelta a la inquietud propia; a tratar de mirar más allá del estricto rigor que se da en las clases.., yo tal vez eso es lo que trato de hacer, de mostrar a los estudiantes, que por una parte los conocimientos que se tienen de los modelos que explican los fenómenos desde el punto de vista científico, pues son válidos hasta cierto punto, tienen ciertas limitaciones, tienen cierto marco de aplicabilidad yo les trato de resaltar eso y que busquen ampliar esos rasgos de aplicabilidad, esos rango donde se aplican los conceptos, para que los usen como más les interesen, yo noto sin embargo que la actitud de los estudiantes, sigue siendo la de ir a escuchar y en últimas ha aprender lo que alguien sabe, que se supone es lo que se debe saber, entonces, yo no lo veo de esa manera, yo lo veo más bien como una ayuda en la cual, el profesor debería mostrarle pues desde su propia perspectiva, los campos en los que se puede ampliar el conocimiento una persona, eso que quiere ampliar lo decide cada persona por sí misma".

Lo anterior, se puede contrastar perfectamente en el vídeo, donde cada estudiante desde su perspectiva conceptual participa activamente en la clase, dialoga con el profesor, se cuestiona, identifica sus errores y sus aciertos conceptuales y da rienda suelta a su imaginación a la hora de representar lo que esta diciendo frente a modelos teóricamente aceptados. Sin embargo, hay un estudiante a quien parece no le interesa el 
tema de la calase o simplemente su nivel de conceptualización esta muy alejado y no logra participar activa y eficientemente en la clase.

Esta visión de la enseñanza y aprendizaje de las ciencias del profesor B y la del profesor A, se asemeja mucho a los modelos vigentes en una didáctica de resolución de problemas de una metodología de las ciencias; obviamente guardando las proporciones y como límite de las dos experiencias observadas, la formación inicial de cada profesor.

En cuanto a la coherencia entre el pensamiento y la actuación del maestro, encontramos que los profesores se describen y actúan de una manera muy semejante, se puede decir que estos profesores tienen un amplio conocimiento sobre su quehacer diario y lo que ellos buscan a través de su trabajo en el aula de clase; esto se ratifica al analizar lo que cada uno de ellos piensa primero de su actuación en el aula y por último la descripción breve del rol de cada uno frente a sus estudiantes, respuestas estas corroboradas una vez los profesores analizaron su propio video de clase. Empecemos por el profesor A:

"Yo, considero que la institución escolar y el profesor, no solamente, deben favorecer el aprendizaje de los contenidos disciplinares, sino también desarrollar cierto tipo de habilidades y capacidades en las personas las que tienen que ver diría yo con el pensamiento creativo y con el pensamiento inteligente entonces, yo considero pues, más que aprender contenidos, lo que se debe favorecer en la institución escolar, es el desarrollo de esas destrezas y esas habilidades, por lo tanto el papel del profesor, es muy importante, debe posibilitar espacios, en términos generales, ambientes donde los niños y los adolescentes puedan desarrollarlas. Yo pienso que esa es una idea fundamental y en eso donde yo realmente he trabajado en los últimos años, en investigaciones que tienen que ver con el desarrollo cognitivo, sobre inteligencia sobre capacidades y destrezas, yo pienso que, si se favorece ese tipo de destrezas se podría lograr que las personas independientemente de que estén o no en un ambiente educativo puedan desarrollarse".

Para el profesor A, quien de paso se ha involucrado en problemas de investigación educativa centrado principalmente en la forma cómo aprenden los sujetos, parecería ser que los resultados de sus investigaciones se reflejan en sus clases, al igual que la sistematización y organización que el profesor tiene de las mismas, la claridad en las preguntas y la forma como maneja las respuestas del auditorio. Lo anterior, permite concluir que el profesor emplea en su práctica docente cotidiana los modelos cognitivos sobre los cuales él investiga. Esto podría considerarse como una ilustración de la aplicación de una práctica investigativa en el aula. Para el profesor $\mathrm{B}$, encontramos que su coherencia y pensamiento dependen principalmente de:

"Debería haber un acuerdo mínimo en que los profesores establezcan cada uno desde su estilo los requisitos con los cuáles van a considerar que un estudiante se promueva a un curso o no, qué tipo de exigencia se van a dar, por ejemplo, hay que cuidar que los estudiantes sean honestos, que si se les asigna una tarea especial, ellos la hacen que fundamentalmente ellos están interesados en aprender algo y el asignarles tareas, es un mecanismo para que ellos se enfrenten a cierto tipo de ejercicios, cierto tipo de problemas y pongan en juego sus habilidades y puedan desarrollar sus conocimientos, de una parte y puedan desarrollar sus propias habilidades, sin embargo, eso en la práctica no se hace". 
El profesor centra su atención, en el interés que los alumnos tienen cuando aprenden nuevos conceptos y en el desarrollo de habilidades para el dominio de conceptos teóricos y prácticos en el campo de las ciencias, así como también de valores humanos como la honestidad.

Según lo anterior, las notas provenientes de la observación del video, la transcripción de la entrevista y el análisis que el profesor realizó de su video, se logra identificar el papel del profesor, su rol, su actuación, desde el cómo se ve él y la actuación misma. Así, para el profesor A,

"Más que preocuparme por mi desempeño en el aula, me preocupan más los niveles de aprendizaje que adquieran los estudiantes, quiero decir que por lo general los profesores que somos novatos o expertos nos preocupamos mucho por nuestro desempeño en el aula, eso hace que pensemos mucho en nuestra actuación, la forma como vamos a presentar los contenidos, en la forma como estoy hablando si estoy presentando bien los contenidos en el tablero, etc. ... en cuanto a las características de la personalidad, me considero un profesor relativamente cordial, dentro de la normalidad, pienso que la dinámica de las clase que tengo, se desarrollan en un ambiente más centrado en el logro de objetivos que en la disciplina en si misma, en cierta medida me considero un profesor tolerante, paciente y en la medida de lo posible justo, en cuanto a mis capacidades cognoscitivas, yo diría que procuro ser organizado en la presentación de los temas, en eso soy bastante incisivo, sin embargo pues no es fácil, porque a veces uno adecua los contenidos disciplinares a la lógica de la disciplina eso no significa pues que los estudiantes de la misma forma aprendan, es decir, siempre hay una incertidumbre entre lo que uno piensa que esta haciendo y lo que realmente esta haciendo, yo diría que esos son como las características que a rasgos generales definen mi trabajo".

En esta descripción o imagen de sí mismo el profesor A, refleja su actuación en el aula de clase. Es así como, a partir una negociación sobre aspectos conceptuales y evaluativos de la clase, el profesor A da inicio a la misma, haciendo énfasis en los conceptos que se han desarrollado anteriormente tanto en la teoría como en las prácticas de laboratorio, en otras palabras, el profesor realiza una recontextualización cognitiva y a partir de las ideas de los alumnos, formula nuevas inquietudes e introduce conceptos e ideas fundamentales para cuestionar a los alumnos y ponerlos a trabajar en grupos.

Algo muy particular de las clases del profesor $A$, es que sus alumnos toman apuntes o notas simultáneamente con sus explicaciones, lo cual trae como consecuencia, como se muestra en el video, que hay puntos durante la explicación del profesor que los estudiantes no entienden, precisamente por la falta de atención, comentario éste que el profesor realiza al observar su vídeo. Esta particularidad se resalta, como diferencia de las clases del profesor B, en donde los alumnos llevan a ellas una serie de apuntes y notas previas, que les permiten participar y realizar conjuntamente con el docente las explicaciones y los aportes al tema tratado. Solamente, al terminar cada sesión, los estudiantes toman sus notas finales. El profesor comenta, que esa estrategia forma parte de la metodología de su clase y por tanto la toma de notas no genera dificultad en el entendimiento de conceptos.

Los dos profesores, tienen un amplio manejo del auditorio, lenguaje químico, interacción permanente entre el discurso de las clases y los conceptos de los estudiantes, la evaluación es permanente, con ejercicios de aplicación, o confrontación y relación entre conocimientos y ambos posibilitan la discusión entre los alumnos como una pequeña 
comunidad de "científicos" debatiendo ideas, conceptos, contrastando hechos o fenómenos y concluyendo. Es así, como el profesor B, considera fundamental en su papel:

"Poseer conocimientos suficientemente amplios y profundos de las ciencias, y de otra parte habilidades para poder comunicar ese conocimiento que tiene... Lo que más me gusta es la oportunidad que se presenta de vez en cuando para que yo mismo pueda buscar digamos modelos más actualizados de lo que trate de ilustrar, lo que más me disgusta es que eso no es muy frecuente. Si digamos, yo me sentiría más a gusto si hubiera un grupo de estudiantes que permanentemente lo obligaran a uno a estar estudiando más, desafortunadamente jos grupos lo que asumen es que uno ya se las sabe todas y ellos esperan que uno les cuente lo que ellos tienen que saberse, desde ese punto de vista es decepcionante la labor de docente en ese plan".

\section{CONCLUSIONES}

El presente estudio de caso nos permite evidenciar las concepciones que sobre la ciencia en general y particularmente la química, tienen dos profesores. De igual manera, se reflexiona en torno al proceso de enseñanza y aprendizaje de las ciencias, las creencias del profesor y el conocimiento del compromiso que tienen sus alumnos en virtud a que son el eje fundamental que soporta la práctica educativa y que, como aquí se demuestra, no inhibe el cambio y evolución de la práctica misma

Muchas podrían ser las reflexiones y análisis que de este estudio de caso se pueden hacer aquí, entre ellas podríamos mencionar, que aunque la formación inicial de los dos profesores es diferente, al igual que su pensamiento y actuación, sus clases se desarrollan desde el paradigma constructivista de la enseñanza y el aprendizaje de las ciencias.

Cada una de las concepciones expresadas por los profesores sobre el cómo conciben la ciencia, los procesos de enseñanza y aprendizaje y su rol en el aula, son elementos fundamentales a la hora de desempeñarse como profesor, máxime cuando su actuación en el aula es ante docentes en formación, de quienes se espera que críticamente analicen la actuación de su profesor y construyan su propio quehacer para desempeñarse en un futuro como educadores.

En este campo de investigación didáctica todavía hay mucho camino por recorrer, las líneas anteriores son un aporte a un campo actual de la investigación educativa y estudios de caso como éste en el campo de la enseñanza de las ciencias y su aprendizaje, son necesarios ya que contribuyen al conocimiento del pensamiento del profesor y sus relaciones con su actuación en aula (Stallings, 1995, p. 31). 


\section{REFERENCIAS}

Fernández, G. y Elortegui, N. 1996. ¿Qué piensan los profesores de cómo se debe enseñar? Enseñanza de las Ciencias 14 (3). 331 -342.

Guerrero, José F. 1987. La investigación etnográfica en el ámbito educativo. Investigación en la Escuela No. 3, España.

Martínez, Jaume, 1988. El estudio de caso en la investigación educativa. Investigación en la Escuela, No. 6. España.

Reyes, L., Salcedo. L. E. y Perafán, G. A. 1999. Acciones y creencias. Tesoro oculto del educador. Universidad Pedagógica Nacional.

Smith, John K. 1983. Quantitative versus qualitative research: An attempt to clarity the issue. Educational Researcher. Marzo, pp. 6-13.

Stallings. William. 1995. Confessions of a Quantitative Educational Researcher trying to teach Qualitative Research. USA. Journal Education Researcher. April, pp. 31-32.

Stenhouse, L. 1990. Case Study Methods. En Keeves, J. P. Educational Research, methodology and measurement, an international. Hand Bock. Oxford Pergamon. Press, pp. 49-53. 